\title{
NONLINEAR DRIFT OF THE SPRING GRAVIMETER CAUSED BY AIR PRESSURE FROM THE KUNMING GS15 GRAVIMETERS
}

\author{
WEI Jin ${ }^{1,2}$ \\ SHEN Wenbin ${ }^{1}$ \\ LI Hui $^{2}$ \\ Liu Ziwei ${ }^{2}$ \\ ${ }^{1}$ School of Geodesy and Geomatics, Wuhan University, Wuhan 430079, China \\ ${ }^{2}$ Key Laboratory of Earthquake Geodesy, Institute of Seismology, CEA, Wuhan 430071, China
}

\begin{abstract}
In order to monitor and correct the meteorological factors of the spring gravity meter, the characteristics of the time varying gravity changes caused by meteorological factors were analyzed. Kunming GS15 gravity meter from 2007 continuous gravity observation has been carried out with the sampling rate of the pressure observation. In this study, we first compare the effects of 4 types of gravity meter and 3 different types of stations on the gravity observed seasonal gravity signals. It is indicated that the observed seasonal gravity signal of the cave is only $1 / 10$, and there is a constant temperature and constant pressure device. Compared with the same time, the gravity signal of the gravity signal is about 100 times smaller. The influence of the pressure load of the gravity meter is tested by using the theory of pressure. The results show that only the actual value of $2 c p d-3 c p d$ pressure load varies from -0.395 to $-0.280 \times 10-8 \mathrm{~ms}^{-2}$, and the $1 c p d$ to $1 \mathrm{cpm}$ periodic partial type gravity meter is also in accordance with the law of gravity and air pressure. And with the characteristics of time lag. In this paper, the nonlinear zero drift parameters of the linear regression model with time lag and the time series of the GS15 gravity meter are used to simulate the nonlinear zero drift parameters of the gravity meter. The results show that the gravity signal contains time lag 35 hours, and the air pressure admittance is $0.8 \times 10-8 \mathrm{~ms}^{-2} / \mathrm{mbar}$. The correlation can reach $79 \%$. The gravity changes signal and satellite gravity as well as the gravity water load signal of the land water model are the same as the gravity water load signal in the autumn as the minimum value, and the seasonal variation of the maximum value of gravity in summer.
\end{abstract}

Keywords: GS15 gravimeter meter, air pressure admittance, nonlinear air pressure correction, superconducting gravimeter

\section{INTRODUCTION}

Kunming GS15 gravity meter has had a digital transformation from the GS metal spring gravimeter[1] which is originally produced by German Askania factory, it has been connected into the digital earthquake observation network to do the continuous gravity observation. When the research on influence of meteorological factors to gravity has been done, pressure has received great attention from both theoretical research and actual observation. Chinese Academy of Sciences had a summary on the relationship between pressure and gravity from the perspectives of theory and actual observation in 1997[2], and in 2008, it has found that gravity and frequency domain between pressure has dependencies in the superconducting gravity observation[3]. In analysis of the LCR-ET20 spring tidal gravimeter, it has found when it is good condition, the pressure and the oceanic gravity signals within the tidal frequency can be effectively recorded, while the proposal of the long-period correction of zero drift caused by creep characteristics of spring need to be paid attention [4, 13]. China Seismological Bureau concluded the Padang M5.1 earthquake occurred in 2013 and found that the observation data from Yichang DZW gravimeter has broken the year rule before the earthquake. It also had analysis on the influencing factors to year cycle change and on the possible factors caused the rule breaking. When having the data analysis, it simply used theory of pressure load to correct the pressure load, having no further consideration on the influence of nonlinear factor caused by pressure load[5]. Therefore, this paper studies 
to use Kunming GS15 gravity meter from 2007 continuous gravity observation has been carried out with the sampling rate of the pressure observation, after comparing the constant temperature and constant pressure system(see Table 1) of 4 types and 5 sets of gravity meter[6-9], the station type and the seasonal factor which can have influence on gravity, on this basis, using the theoretical air pressure admittance in the air pressure load theory to study the nonlinear characteristics of different period observation data responded to air pressure. With the consideration of time lag, using Kunming GS15 gravimeter observation data as example, constructing the nonlinear correction model of air pressure load to have nonlinear-factor correction, providing a new research and analysis method for using spring gravimeter to analyze the time-varying gravity change characteristics in local area caused by non-meteorological factors.

\section{COMPARATIVE ANALYSIS OF CONTINUOUS GRAVITY OBSERVATION AND INFLUENCE OF AIR PRESSURE LOAD}

The relationship between gravity observation data and air pressure change should conform to the theoretical correlation referred in reference [2-3]. Therefore this paper firstly have processed at least 4-year observation data from Jiufeng seismostation (DZW) gravimeter,academy of sciences Jiufeng station SG superconducting gravimeter, Zhangjiakou gphone gravimeter, Nnshan GS15 gravimeter and Kunming GS15 gravimeter, using the gravity residual time series corrected by using theoretical gravity earth tide to study the same-address observation data of air pressure and its load influence rule.

\section{DATA PRE-PROCESSING MODEL}

When the continuous gravity observation data has been processed, adopting the elimination - recovery data preprocessing method to eliminate all factors which will interfere the earth tide observation include jump error, drift, error data, seismic vibration caused by the earthquake. Meanwhile, using model (1) in the elimination process of the above preprocessing to simulate gravity residual time series which includes: formation process of the spring relaxation, change of the linear shift before and after the machine failure and the nonlinear drift of the spring gravimeter caused by drift or other causes before and after the failure, and correct them[10,11]. Figure 1 is the pre-processing with Kunming GS15 gravimeter as asn example.

$$
\begin{aligned}
& g_{\text {res }}\left(t_{i}\right)=g_{\text {untinear }}\left(t_{i}\right)+v_{i}=d_{0}+d_{1} t_{i}+\sum_{j=1}^{N_{\text {ab }}}\left(a_{j} \cos \left(\frac{2 \pi}{T_{j}} t_{i}\right)+b_{j} \sin \left(\frac{2 \pi}{T_{j}} t_{i}\right)\right)+\sum_{j=1}^{N_{g}} g_{j} H\left(t_{i}-T_{g j}\right) \\
& +\sum_{j=1}^{N_{k}} h_{j} H\left(t_{i}-T_{h j}\right) t_{i}+\sum_{j=1}^{N_{k}} k_{j} \exp \left(-\left(t_{i}-T_{k j}\right) / \tau_{j}\right) H\left(t_{i}-T_{k j}\right) t_{i}+v_{i}
\end{aligned}
$$

Among them: $t_{i}$ is the sample epoch. Here the unit is minute; $g_{\text {res }}\left(t_{i}\right)$ is the gravity residual time sequence after the tidal correction; $H$ is the step function; $d_{0}$ is the constant term of the residual time sequence; $d_{1}$ is the linear term of the gravity observation data; parameter $a_{j}, b_{j}$ are simulated annual or seasonal load factors in the residual by using sinecosine function. $g_{j}$ is the jump error caused at time $T_{g j}$. When $T_{g j}$ is the seismic moment, $g_{j}$ is the coseismic gravity change. $h_{j}$ is the gravity change rate after the earthquake. $k_{j}$ is the amplitude of spring's relaxation process simulated by using exponential function after the earthquake; $\tau_{j}$ is the relaxation time of spring gravimeter after the earthquake.

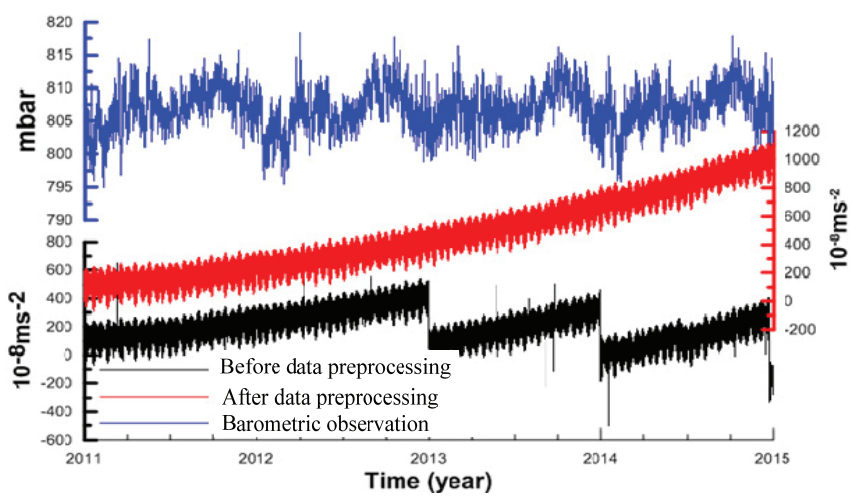

Fig.1 Comparison of Kunming GS15 gravimeter before and after the data pre-processing

Note:The comparison of observation time sequence before and after the data pre-processing shows no significant jump or drift observation data. The color of the longitudinal axis is same with the corresponding color of the observation sequence. The air pressure load influence of Kunming station shows seasonal characteristics. While the gravity earth tide observation appears about 100 $200 \times 10^{-8} \mathrm{~m} \cdot \mathrm{s}^{-2} /$ achange rate and the increasing zero drift.

\section{COMPARATIVE ANALYSIS OF DIFFERENT TYPE GRAVIMETER OBSERVATION AND INFLUENCE OF AIR PRESSURE CHANGE}

According to the continuous gravity observation data from the above 4 types and 5 sets gravimeters, having eliminationrecovery method to pre-process the data. And all adopting gravity earth tide theoretical value to have tidal correction and have zero drift correction to polynomial which is not more than 3 steps. Comparing the residual time series with the air pressure (see as Figure 2). Meanwhile, combining the analysis result in Table 2 with the gravimeter station and the devices information to have a comparative analysis (see Table1).

The results showed that among the 5 gravimeters: the seasonal changing amplitude of gravity residual of the gravimeter which used constant temperature device almost only $\pm 10 \times 10^{-8} \mathrm{~m} \cdot \mathrm{s}^{-2}$. Which the two types of gravimeter (GS15 and DZW) which didn't use the constant temperature device will change according to the change of the observation station type. Among them, the smallest seasonal change of gravity residual is the cave $\left( \pm 10 \times 10^{-8} \mathrm{~m} \cdot \mathrm{s}^{-2}\right)$, the biggest one is the earth surface $\left( \pm 1200 \times 10^{-8} \mathrm{~m} \cdot \mathrm{s}^{-2}\right)$. If the constant temperature device is the reduced pendulum causes the nonlinear drift 
of the spring because of the change of temperature or air pressure, cave observation plays a role of the above constant temperature effect at some degree. Thus, if using gravity residual seasonal change as the measurement of judge the gravimeter observation quality, its size is not only related with the constant-temperature device, but also related with the observation types. The comparison of 4 sets of different type of continuous gravity observation gravity residual time series and the air pressure change is shown as Figure 2.

Tab.1 Comparison of observations and air pressure of gravity meter

\begin{tabular}{|c|c|c|c|c|c|}
\hline \multirow[b]{2}{*}{ 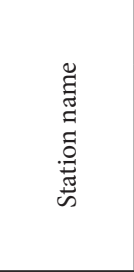 } & \multirow[b]{2}{*}{$\begin{array}{l}0 \\
0 \\
0 \\
0 \\
0 \\
0 \\
0 \\
0 \\
0 \\
0 \\
0 \\
0 \\
0 \\
0 \\
0 \\
0\end{array}$} & \multirow{2}{*}{ 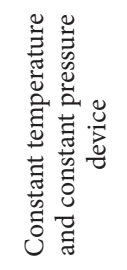 } & \multirow[b]{2}{*}{ 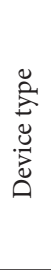 } & \multicolumn{2}{|c|}{$\begin{array}{c}\text { Seasonal change of gravity } \\
\text { residual }\end{array}$} \\
\hline & & & & 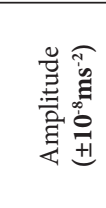 & 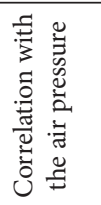 \\
\hline Kunming & Cave & - & $\frac{n}{\overrightarrow{3}}$ & 10 & + \\
\hline $\begin{array}{c}\text { Academy } \\
\text { of sciences } \\
\text { Jiufeng } \\
\text { station } \\
\end{array}$ & Basement & Dewar & 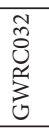 & 10 & - \\
\hline Panzhihua & $\begin{array}{c}\text { Semi- } \\
\text { basement }\end{array}$ & - & $\stackrel{n}{\mathscr{L}}$ & 50 & + \\
\hline $\begin{array}{c}\text { Jiufeng } \\
\text { earthquake } \\
\text { station }\end{array}$ & $\begin{array}{l}\text { earth } \\
\text { surface }\end{array}$ & - & 芯 & 1200 & - \\
\hline $\begin{array}{c}\text { Zhangjiakou } \\
\text { earthquake } \\
\text { station }\end{array}$ & Cave & $\begin{array}{l}\text { Constant } \\
\text { temperature } \\
\text { air bag }\end{array}$ & $\begin{array}{l}\stackrel{P}{\frac{1}{2}} \\
\frac{1}{00}\end{array}$ & 10 & + \\
\hline
\end{tabular}

Note: gPhone uses constant temperature and constant pressure air bag to work, in order to insure that the observation of metal spring of the device maintains in the smallest change of environment [08]; the superconducting ball
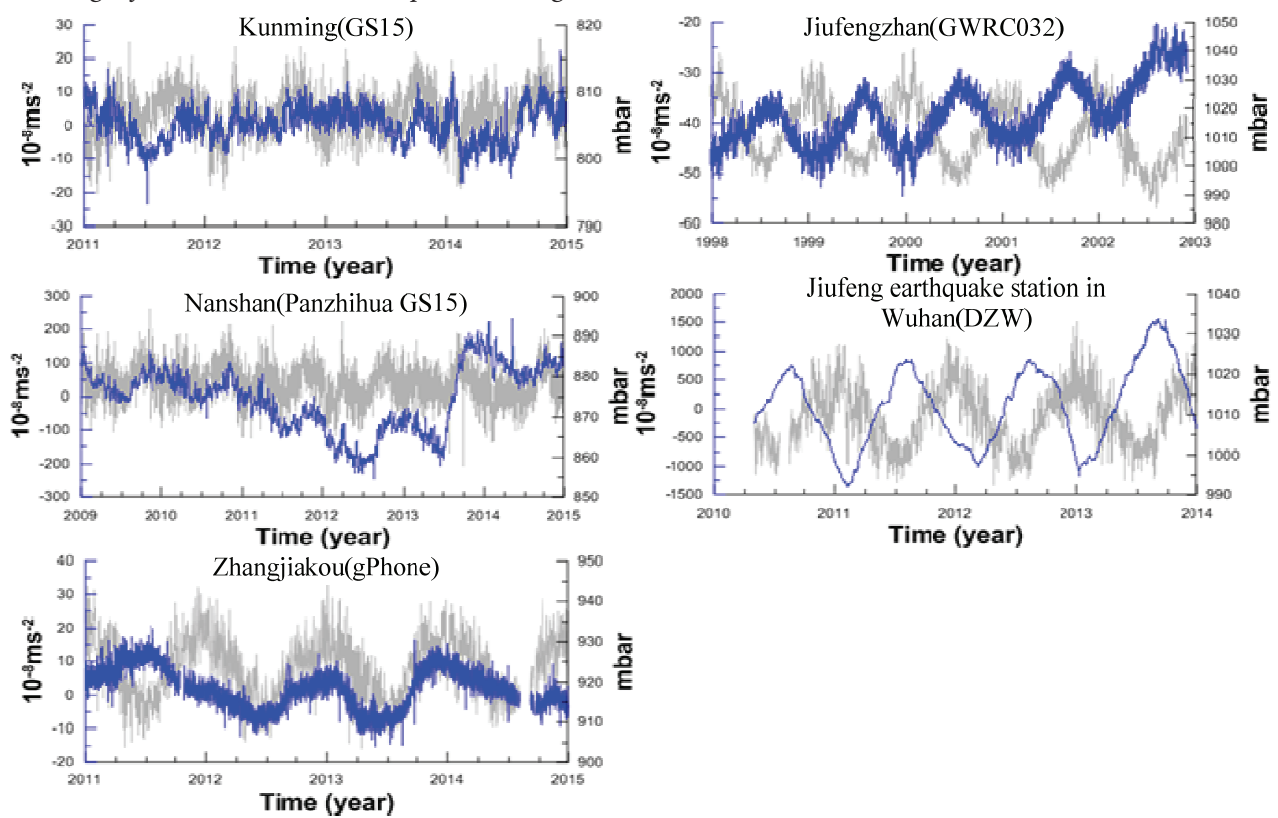

Fig. 2 Comparison of 4 sets of different type of continuous gravity observation gravity residual time series and the air pressure change

Note: In the figure the gray time series is the air observation of the continuous gravimeter same-addressed observation; blue time series is the gravity residual time series of the continuous gravity observation; of the GWR gravimeter works in the Dewar bottle (constant temperature, almost vacuum)[09]; gPh70 indicates the No.70 device of the gPhone gravimeter. The correlation with the air pressure “+” indicates positive correlation, “-” indicates negative correlation.

The academy of sciences Jiufeng station and the Jiufeng earthquake station which are all not belong to the cave observation and located less than $5 \mathrm{~km}$ away from each other, their gravity residual time series shows that: the observation quality with the constant temperature device (dewar) is 100 times than which without the constant temperature device. While the same type, both with no constant temperature device 2 sets of GS15 gravimeters, since the different observation station type, their annual changing amplitude of the different observation data only different between 5 to 10 times (cave type is better than that of half basement type). According to the comparison of the data quality different times, the effect of controlling the constant temperature device is much more greater than the effect of the observation station type (almost 20 times).

From the rule of the annual cycle changing rule of the gravity residual time series and the air pressure: Wuhan Jiufeng area 2 sets of gravimeters are all presented the negative correlation characteristics which is the same changing direction with pressure load influence. While the other 3 sets of gravimeters showed the positive correlation characteristics. Based on the disagree of the above two kinds of observation results and the air pressure load theory results[2-3], this paper will analyze the relation of different time scale between gravity observation and the air pressure load influence. 


\section{ANALYSIS OF RELATIONSHIP BETWEEN GRAVITY OBSERVATION AT DIFFERENT TIME AND PRESSURE LOAD}

The pressure load theory shows that [2], the changing of air pressure and gravity should be negative correlation. The magnitude of the air pressure admittance is changing from - 0.395 to $-0.280 \times 10^{-8} \mathrm{~m} \cdot \mathrm{s}^{-2}$. The above 4 types and 5 sets of devices' annual periodic change is not completely consistent with the air pressure load theory. Therefore, this paper uses VAV harmonic analysis method [6] to analyze the air pressure load influence within $1-7 \mathrm{cpd}$ frequency range, while the air pressure admittance of 3 days, 1 week, 2 weeks, 1 month, 1 season, 0.5 year, 1 year uses gravity residual and air pressure sequence of linear regression to obtain. The analysis results as shown in Figure 3.

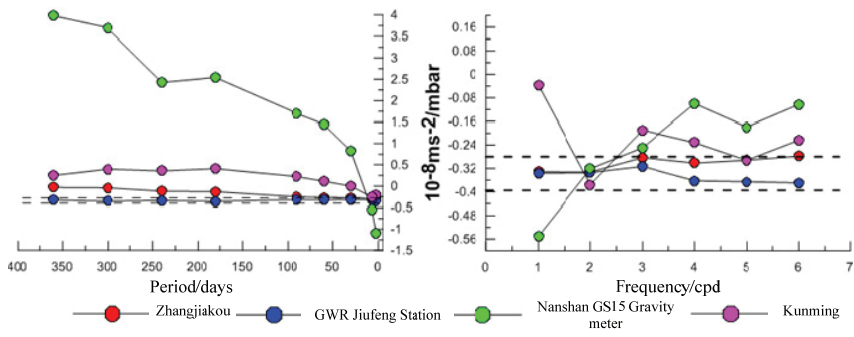

Fig.3 Relationship between gravity observation at different time and pressure load
From the above analysis results of 4 sets of devices show that, the superconducting gravimeter, no matter its 1 year scale change or its admittance relationship between air pressure and gravity within $1 / 6$ daily wave frequency range is all changing within the theoretical value scope. While in the analysis of spring gravimeter less than 1 day periodic cycle, change of air pressure and gravity mainly be negative correlation, the observation sequence within $2 \mathrm{cpd}$ and $3 \mathrm{cpd}$ frequency scope basically conforms to the pressure load theory. Other frequency scope almost pass the scope of the theoretical simulation. The observation data which more than the 1 day periodic cycle only within 1-2 weeks (gPhone gravimeter about 100 days) the observation sequence still conform to the air pressure load theory. A longer time-span observation data show that the changing characteristics of air pressure admittance increasing. Among them, gPhone gravimeter changes slowly, the Nanshan GS15 which located in semibasement and without constant temperature device changes in fastest way. Furthermore, the air pressure load influence of long term gravity observation data is far greater than the theoretical observation value, and with the phenomenon of time delay.

\section{THE NONLINEAR PRESSURE CORRECTION AND CHARACTERISTIC STUDY OF KUNMING GS15 GRAVIMETER}

From the above different observation environment,
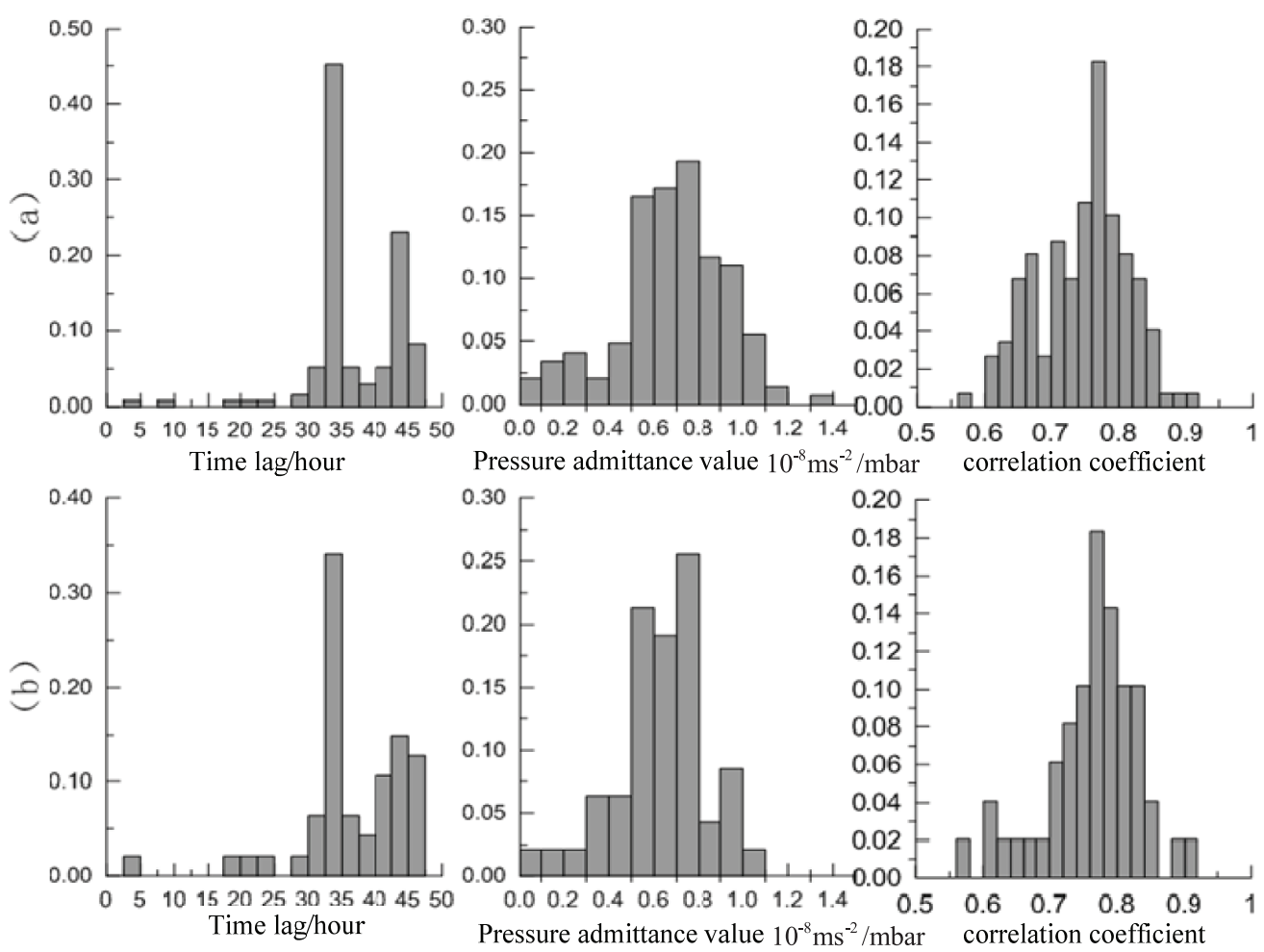

Fig.4 Study on the statistical characteristics of the influence parameters of different time scale air pressure

Note: (a) is the statistics of calculation results of observation data between 1 month to 1 year time span; (b) is the statistics of calculation of time delay, air pressure admittance and related coefficient of 1 month observation data; vertical coordinates are all ratio which is the corresponding statistical samples account for the total calculation samples. 
observation device, time scale to study the rule of influence of pressure to the gravity, indicating that the pressure load scale produced by the air pressure to the spring gravimeter will gradually increase, and with some nonlinear changing characteristics such as time delay and so on. In order to obtain the gravity change of the spring gravimeter caused by non-pressure elements, choosing the station observation environment, data observation quality, with same-value meteorological observation Kunming GS15 gravimeter's observation data, with the consideration of phase lag characteristics to have correction of nonlinear air pressure load influence and characteristics study.

\section{NONLINEAR AIR PRESSURE CORRECTION MODEL AND ANALYSIS}

Due to the air pressure has different influence situation to the different gravity observation time sequence, and accompanied by the characteristics of the phase lag. This study chooses different time scale, different time lag characteristics and adopts the following model to have the statistical analysis, the results show:

$$
\Delta g_{i}=a \Delta P_{i+\Delta t}+b+c t_{i+\Delta t}
$$
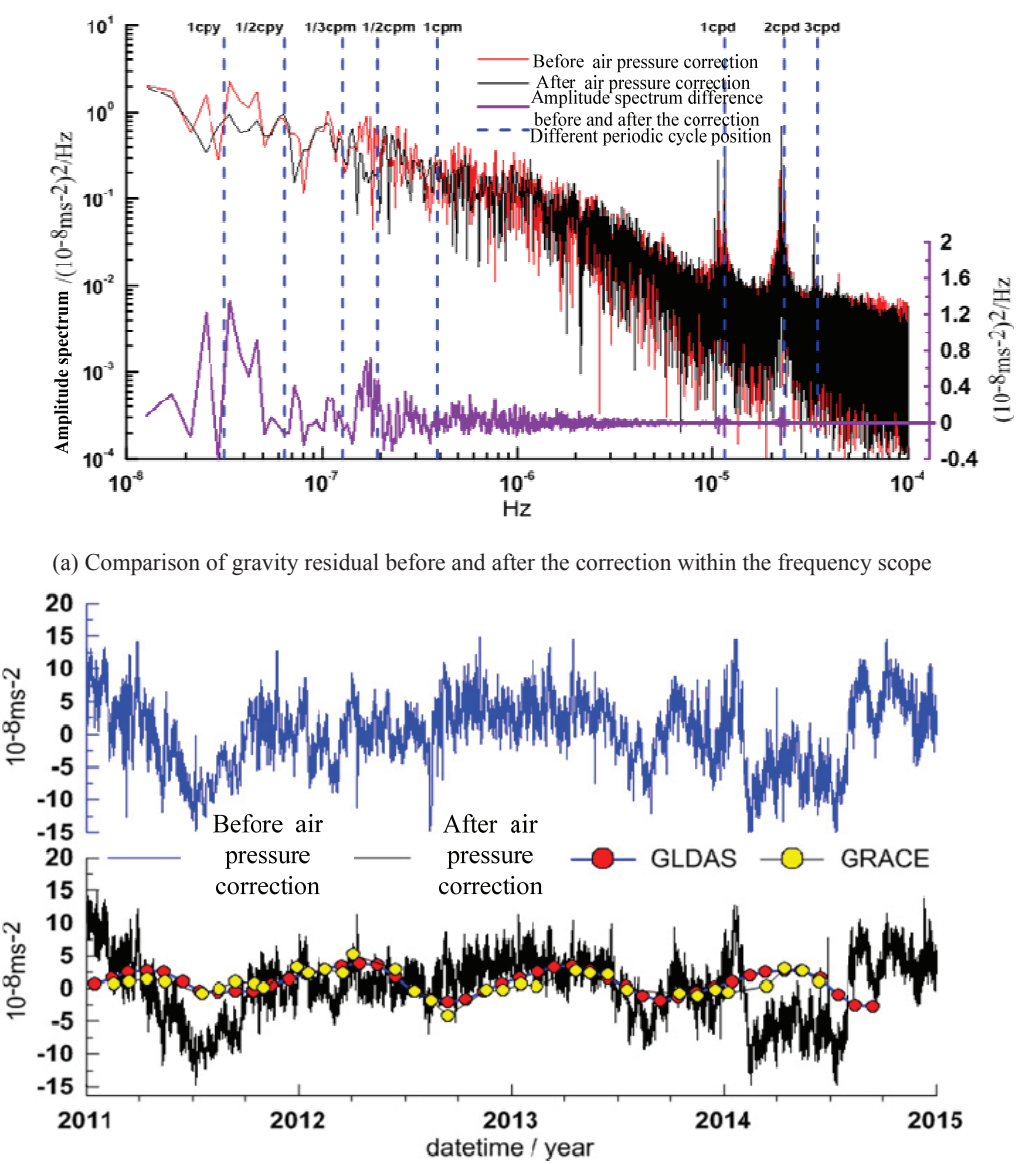

(b) Comparison of gravity residual before and after the correction within the time scope Fig.5 Comparison of time series and spectral analysis results of nonlinear pressure corrections for gravity residuals

Note: cpy, cpm, cpd are respectively short for cycle percent year, cycle percent month, cycle percent day. The blue dotted line indications in the figure are positions of frequency respectively is $1 c p d$, $2 c p d, 3 c p d, 1 / 3 c p m, 1 / 2 c p m, 1 c p m, 1 / 2 c p y, 1 c p y$
Among which: $\Delta g_{i}$ is the gravity residual time sequence, $a$ is the air pressure admittance, $b$ is the pressure time-varying change constant, $\mathrm{c}$ is the pressure time-varying change firstdegree item. $\Delta t$ is the time delay. $\Delta P$ is the pressure changing value.

The statistical results show that, about $45 \%$ statistical results show there exists 35 hours phase lag between gravity and air pressure; the range scope of air pressure admittance is $0.6-1.0 \times 10^{-8} \mathrm{~m} \cdot \mathrm{s}^{-2}$; Above $45 \%$ statistical records show that the correlation between gravity and air pressure is above $80 \%$. Therefore, this paper adopts 35 hours phase lag, air pressure nonlinear load influence $0.8 \times 10^{-8} \mathrm{~m} \cdot \mathrm{s}^{-2}$ to correct Kunming GS15 gravimeter received air pressure nonlinear load influence.

\section{COMPARISON AND ANALYSIS OF NONLINEAR AIR PRESSURE CORRECTION}

By using the above model to have pressure correction to Kunming station gravity residual time series. Using amplitude spectrum analysis method to compare the amplitude spectrum difference before and after the correction (as shown in Figure 5-a). The result shows: the tide of gravity residual (1cpd 3cpd) signal's amplitude is less than long period $(1 \mathrm{cpm} \sim 1 \mathrm{cpy})$. The difference value of gravity residual signal amplitude spectrum before and after the correction shows: annual period signal (1cpy $\sim 1 / 2$ cpy) and seasonal period signal $(1 / 3 \mathrm{cpm} \sim 1 / 2 \mathrm{cpm})$ decrease. Annual period decreases $1.4 \times\left(10^{-8} \mathrm{~m} \cdot \mathrm{s}^{-2}\right)^{2} / \mathrm{Hz}$, seasonal period signal decreases $0.6 \times\left(10^{-8} \mathrm{~m} \cdot \mathrm{s}^{-2}\right)^{2} / \mathrm{Hz}$, signal which less than $1 \mathrm{cpm}$ period shows no decreasing characteristic. Only the period at $1 \mathrm{cpd}, 2 \mathrm{cpd}$, 3 cpd signals only decrease. Thus it can be seen, the nonlinear influence of pressure's long period mainly effects the annual and seasonal period signal of the gravity observation. For the period less than $1 \mathrm{cpm}$ has no significant influence.

The residual time series used pressure to have nonlinear zero drift correction (as shown in Figure 5-c), the comparison with that before the correction (Figure 5-b), the observed gravity changing details are more obvious. Comparing the above pressure-corrected gravity residual time series with the gravity change caused by this area water-loaded seasonal factor which is simulated by satellite gravity (Grace) and global land water model (Gldas) [11, 12], the result shows that the above two all reach the smallest gravity in fall in 2012, 2013, while reaching the biggest in summer, this is the seasonal changing characteristics. The inconsistency of magnitude of the seasonal load influence may be the result of simulation model which simulated the largest space scale, it is not completely consistent with the gravity change of Kunming station Local area. 


\section{CONCLUSION AND DISCUSSION}

This paper firstly uses non-tidal analysis method to deal with and compare 4 types and 5 sets of continuous gravimeters' over 4-year pre-processing gravity earth tide observation data, using gravity residual annual and seasonal change as the indicator to measure the observation quality of gravimeter, it found that the size of seasonal change is not only related to the observation station type, but also related with the constant temperature device. The data quality with the precised constant temperature device is about 100 times than the device without the constant temperature device. The comparison of observation station type shows: cave station observation is 5-10 times than the earth surface observation.

For the pressure signal which is the most significant one in the above gravity seasonal change, the study found that 4 types spring gravimeters' observed gravity signal within 2 cpd to 3 cpd frequency scope the observation sequence conform to the pressure load theory. The gravity signal within 1 month also presents negative correlation characteristic with the meteorology, a longer periodic cycle pressure load influence is far greater than the theoretical observation value, and accompanied with the phenomenon of time delay. In order to eliminate the influence of the above factors, taking Kunming GS15 gravimeter whose observation environment is better and assistant observation is complete as example, using the linear regression formula with time delay to analyze the gravity residual time series and the pressure sequence of the same-addressed observation, it has found that gravity signal of Kunming GS15 contains time lag 35 hours, and the air pressure admittance is $0.8 \times 10^{-8} \mathrm{~ms}^{-2} / \mathrm{mbar}$ nonlinear zero drift.

Through the above model to correct the gravity residual time series, comparing the frequency scope characteristics and the time span characteristics of the gravity residual time series signal before and after the correction, within the frequency scope, Kunming station's nonlinear pressure corrected the annual period signal and seasonal period signal in the gravity signals (decreased $1.4-0.6 \times\left(10^{-8} \mathrm{~m} \cdot \mathrm{s}^{-2}\right) 2 / \mathrm{Hz}$ ), the earth tide frequency has no significant correction. Within the time span: the details of the gravity changing are more obvious. The gravity changes signal and satellite gravity as well as the gravity water load signal of the land water model are the same as the gravity water load signal in the autumn as the minimum value, and the seasonal variation of the maximum value of gravity in summer. Due to the regional gravity field change and the difference of the local gravity field, the different scale between the two has been resulted.

\section{REFERENCE}

1. Wei Jin, Shen Chongyang, Liu Shaoming, Dai Miao. Analytic study on abnormal change in time - variable gravity at Yichang seismostation before the M5.1 Badong earthquake. Geodesy and Geodynamics, 2014, 5 (1) : 55-63
2. Xu jianqiao. The theory and analysis of gravity tidal superconducting gravimeter data analysis and processing in Wuhan station[D]. Institute of Geodesy and Geophysics Chinese Academy of Sciences, 1997

3. Mahmoud Abd El -Gelil, et al. Frequency - dependent atmospheric pressure admittance of superconducting gravimeter records using least squares response method [J]. Physics of the Earth and Planetary Interiors, 2008, (170): 24-33

4. Sun Heping, Chen Xiaodong, Liu Ming, etc. Analysis and Comparison of the Tidal Gravity Observations Obtained with LCR-ET20 Spring Gravimeter [J]. ACTA SEISMOLOGICA SINICA, 2002, 24 (5) : 510-515

5. Wei J, Li H, Liu Z W, et al. Observation of superconducting gravimeter at Jiufeng seismic station. Chinese J. Geophys. (in Chinese), 2012, 55(6): 1894- 1902, doi: 10. 6038/j. issn. 0001- 5733. 2012.06. 010.

6. Kang Kaixuan, Li Hui, Peng Peng, Hao Hongtao, Wei Jin. Seasonal variations in hydrological influences on gravity measurements using gPhones[J]. Terr. Atmos. Ocean. Sci., 2011, 22(2): 157-168, doi: 10.3319/Tao. 2010. 08. 02. 01(TibXS)

7. gPhone/PET gravimeter Manual (V1). gMonitor User's Manual, 2008: gMonitor Gravity Data Acquistion and Processing Software

8. GWR Superconducting Gravimeter Manual GWR (superconducting gravimeter) User 's Mannual 2008

9. Wei Jin, Zhao Bin, Tan Hongbo, Yu Dan, Shen Chongyang, Li Hui. Detection of a half-microgal coseismic gravity change after the Ms7.0 Lushan earthquake. Geodesy and Geodynamics, 2013, 4(3):7-11.

10. Wei jin, Li hui, Liu Ziwei, Hao Hongtao, Kang Kaixuan. Study on influence of pressure on gravitymeter SGC053 by use of product spectra[J]. 2011, 31(4):47- 51.

11. David Crossley, Jacques Hinderer, Umberto Riccardi. The measurement of surface gravity[J]. Reports on Progress in physics, 2013, 76(046101)47pp, doi: 10. 1088/0034-4885/76/4/046101

12. Zhou Xin, Sun Wenke, Zhao Bin, et al. Geodetic observations detecting coseismic displacements and gravity changes caused by the $\mathrm{Mw}=9.0$ Tohoku-Oki earthquake[J]. J Geophys Res, 2012, 117: B05408. Doi: 10. 1029/2011JB008849

13. Wang L S, Chen C, Zou R, et al. Using GPS and GRACE to detect seasonal horizontal deformation caused by loading of terrestrial water: A case study in the Himalayas. Chinese 
J. Geophys. (in Chinese), 2014, 57(6): 1792-1804, dio: 10. 6038/cjg20140611

14. Cai, C (Cai, Chuang); Cai, XY (Cai, Xinyong); Li, Y (Li, Yi). MODEL TESTS FOR SHALLOW-WATER SHIP MANEUVERABILITY IN THREE GORGES RESERVOIR [J]. POLISH MARITIME RESEARCH. 2015, 22(2): 136-140.

Fund Project: Natural Science Foundation of China (NSFC) (41204058), China Seismological Bureau Earthquake Research Institute Director Fund (IS201326123)

\title{
CONTACT WITH THE AUTHOR
}

\author{
Wei Jin
}

Key Laboratory of Earthquake Geodesy Institute of Seismology CEA, Hongshan side road No 40 Wuhan 430071

CHINA 